\title{
Cervical spondylophytes causing recurrent and phrenic nerve palsy
}

\author{
Pazardzhikliev Dimitar Dimitrov \\ UMHAT St. George, ENT dept. Plovdiv, BULGARIA
}

\begin{abstract}
Excessive cervical osteophystosis is reported to cause upper aerodigestive tract problems by simple pharyngeal compression, laryngeal oedema or recurrent nerve palsy. We describe a patient presenting with stridor and obstructive apnoea. On physical examination retropharyngeal bulging abutting the epiglottis was found, however endoscopic examination revealed unilateral vocal cord paralysis and hyperplasia of the arytenoid mucosa collapsing into the laryngeal airway on inspiration and contributing to the stridor phenomenon. On chest $\mathrm{x}$-ray a paralysis of the contralateral diaphragm was found. This is an unusual cause of a multi-level obstruction and multiple nerve paralysis. Such concurrent findings have not been published.
\end{abstract}

\section{Introduction}

Cervical ospteophytes on the anterior side of vertebrae are causing upper aerodigestive tract symptoms. ${ }^{1,2}$ Most common is dysphagia, followed by stridor and obstructive sleep apnoea, and rarely acute inspiratory apnoea necessitating a tracheostomy. ${ }^{3}$ Neck pain, globus sensation ad haemoptysis are also reported. ${ }^{4}$

Pathogenetic mechanism is either direct mechanical compression (narrowing the oesophageal or pharyngeal lumen and presenting as dysphagia or abutting the epiglottis and causing stridor, OSAS or dypnoea) or compression and paralysis of the recurrent laryngeal nerve causing dysphonia and acute airway obstruction. 5,6

Most of the reported cases of cervical osteophytes presenting with upper aerodigestive tract symptoms report on unilevel obstruction and straightforward pathogenic mechanism of luminal compression or recurrent paralysis. We hereby report on a multilevel compression and complex pathogenesis of complaints.

\section{Case}

A 72 y.o. male suffering from cerebral palsy was referred to ENT department by his relatives who reported of stridor that was exacerbated on physical strain and emotional stress as well as on apnoeic episodes during sleep. Verbal communication was difficult and it was not possible to elicit symptoms of dysphagia, globus sensation or dysphonia. However, patient presented with inspiratory stridor, but no other signs of acute airway obstruction.

Laryngeal mirror examination showed retropharyngeal mass abutting the epiglottis that vibrated on inspiration and produced the characteristic sound. Rest of the larynx could not be observed. A 30 degree $4 \mathrm{~mm}$ rigid endoscope 
was used in attempt to visualize the rest of the larynx (Fig 1). After it was passed by the epiglottis a right

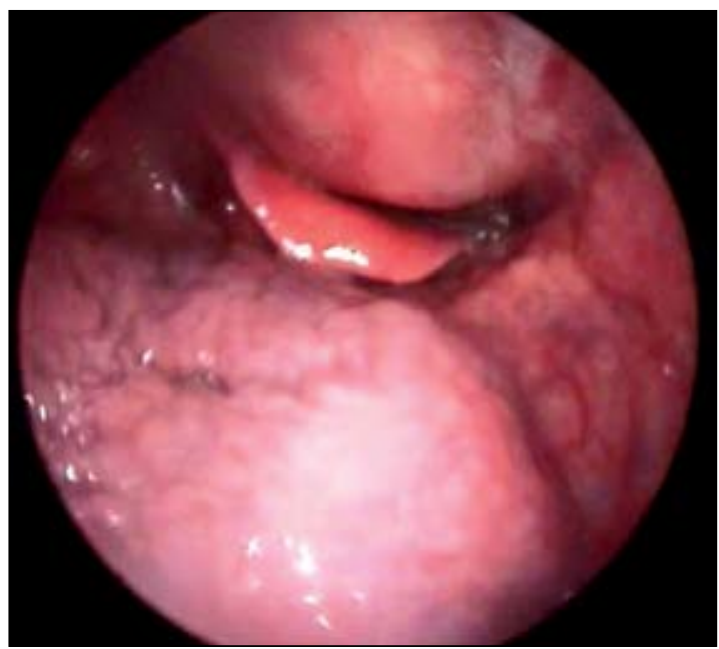

Fig 1 Retropharyngeal cervical osteophytes abutting the epiglottis

sided vocal cord paralysis was established (Fig 2). Besides that the mucosa in the arythenoid region appeared swollen and oedematous and collapsed into

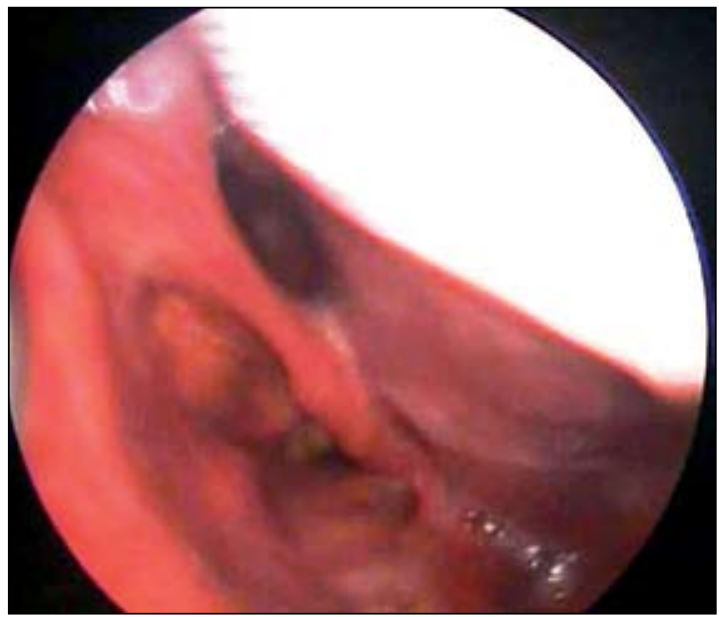

Fig 2 Larynx on inspiration: right vocal cord paralysis and collapse of arytenoids mucosa into the airway (arrows)

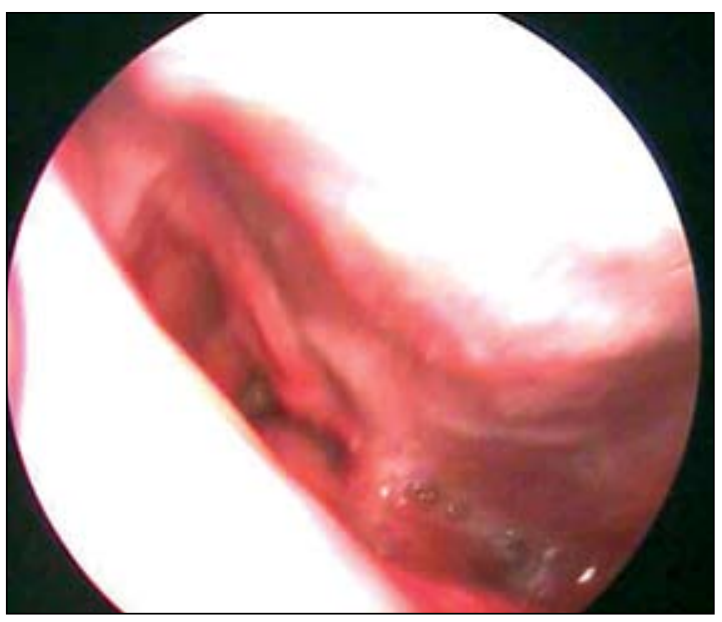

Fig 3 Larynx on expiration: arytenoid mucosa vibrating on expiration the laryngeal lumen during inspiration and vibrated on expiration (Fig 2, 3). A chest $\mathrm{x}$-ray, to our amazement, showed a paralysis of the left diaphragm (Fig 4). CT

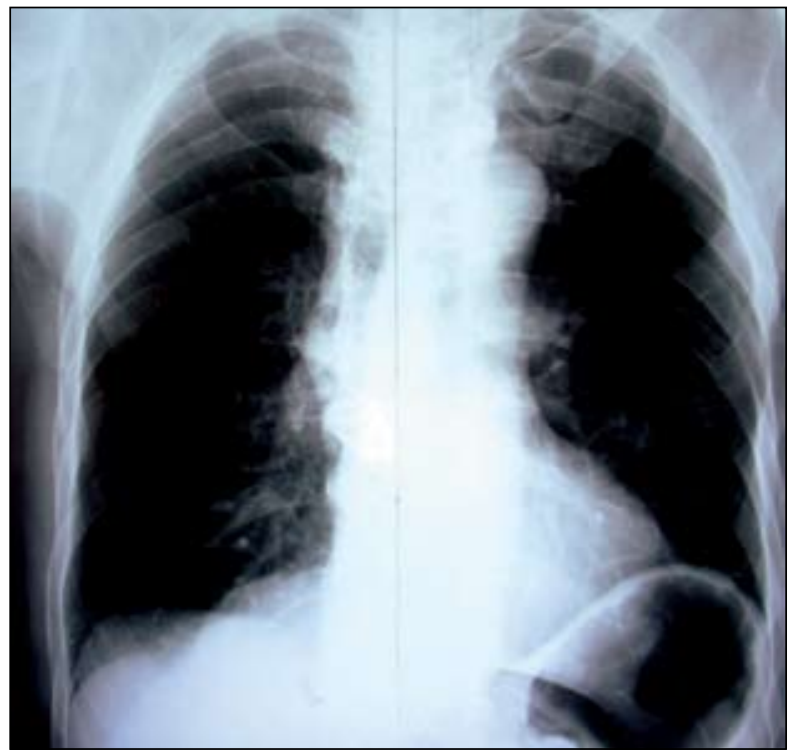

Fig 4

of the neck was performed and showed prominent osteophytes with marked compression of the posterior pharyngeal wall and in the region of the entrance of the right recurrent into the larynx (Fig 5, 6).

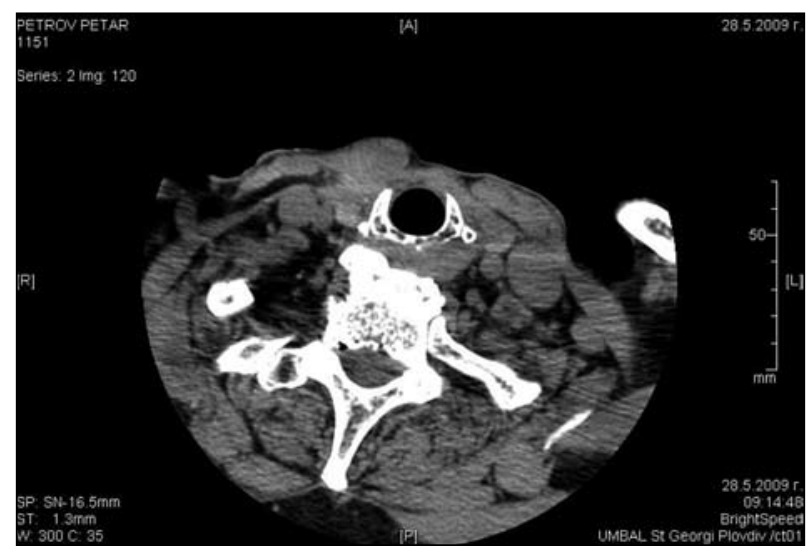

Fig 5

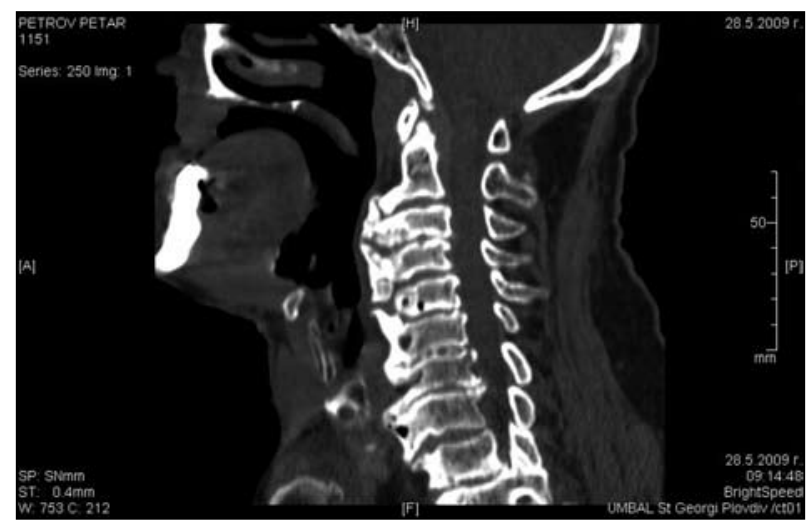

Fig 6 
The patient and his relatives were counseled on the nature of his complaints and the option for surgical treatment of cervical osteophytosis. However, he denied any surgical treatment whatsoever. Since there was no airway compromise necessitating urgent handling the patient was discharged and put on follow-up. Two years after his presentation there is no progression of his complaints.

\section{Discussion}

Cervical osteophytes, as part of Forestier's disease or not, can cause upper aerodigestive tract symptoms when located on the anterior part of vertebrae. Most commonly symptoms are due to simple compression of the neighboring structures and usually present with dysphagia by narrowing of the digestive tract, or with stridor and OSAS by abutting to the epiglottis. However, vocal cord paralysis had been documented, pathogenesis of which had been attributed to retrocricoid compression, inflamation and ulceration. ${ }^{6}$

In the very case that we present at hand are both pathogenic mechanisms: retropharyngeal mass abutting the epiglottis and unilateral vocal cord paralysis due to retrocricoid compression that can be seen on the CT (fig. 6). Such a combination had already been published and necessitated a tracheostomy due to bilateral vocal cord paralysis. ${ }^{6}$ Luckily, in our case there was unilateral paralysis and no airway obstruction. On physical efforts however this unilateral paralysis may have necessitated greater breathing effort and may have precipitated stridor.

Besides that, we could witness oedema of the arythenoid mucosa that collapsed into the airway on inspiration and contributed to the stridor and OSAS and has not been reported previously. The latter we would like to attribute to prominent retrocricoid compression and mechanical irritation, but could also speak of laryngeal reflux precipitated by OSAS triggered by cervical osteophytes. No acid probing was done however. Nevertheless, these findings speak of a multilevel and complex pathogenesis of this patient's complaints that has only been rarely published before.

Accompanying paralysis of the left diaphragm that was found in this case could be attributed to the fact that the phrenic nerve arises from $\mathrm{C} 4$ with contribution from $\mathrm{C} 3$ and $\mathrm{C} 5$. Such a concurrence in cases of cervical osteophytes presenting with upper aerodigestive tract symptoms we could not find in the literature. Although in this very case it was not clinically significant it must be kept in mind in cases that present with respiratory distress.

\section{Conclusion}

The presented case is a nice example that cervical akylozing osteophytes my present with diverse upper aerodigestive tract symptoms of complex and multilevel pathogenesis.

\section{Reference}

1 Brandenberg G, Leibrock LG. Dysphagia and dysphonia secondary to anterior cervical osteophytes. Neurosurgery. 1986 Jan; 18(1): 90-3.

2 Benhabyles M, Brattström H, Sundén G. Dysphagia and dyspnoea as complications in spondylarthritis ankylopoetica with cervical osteophytes. Acta Orthop Scand. 1970; 41(4): 396-401.

3 Papakostas K, Thakar A, Nandapalan V, O'Sullivan G. An unusual case of stridor due to osteophytes of the cervical spine: (Forestier's disease). J Laryngol Otol. 1999 Jan; 113(1): 65-7.

4 Fuerderer S, Eysel-Gosepath K, Schröder U, Delank KS, Eysel P. Retro-pharyngeal obstruction in association with osteophytes of the cervical spine. J Bone Joint Surg Br. 2004 Aug; 86(6): 837-40.

5 McCafferty RR, Harrison MJ, Tamas LB, Larkins MV. Ossification of the anterior longitudinal ligament and Forestier's disease: an analysis of seven cases. J Neurosurg. 1995 Jul; 83(1): 13-7.

6 Aydin K, Ulug T, Simsek T.Case report: bilateral vocal cord paralysis caused by cervical spinal osteophytes. Br J Radiol. 2002 Dec; 75(900): 990-33.

За кореспонденция:

Pazardzhikliev Dimitar Dimitrov

e-mail:dimpaz@abv.bg

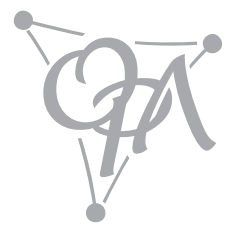

